\title{
Stammes- und Religionsgemeinschaften im mittelalterlichen Südarabien
}

Von Eirik Hovden und Daniel Mahoney

Der Begriff Südarabien bezeichnet die Südwestspitze der Arabischen Halbinsel, die im Gegensatz zu den meisten anderen Regionen Arabiens aufgrund der hohen Berge und der Nähe zum Indischen Ozean mit seinen Monsunregen sehr fruchtbar ist. Eine Definition im weiteren Sinne ist einfach, es handelt sich um ein Gebiet mit großer Bevölkerungsdichte und Landwirtschaft, begrenzt durch das Rote Meer im Westen, den Golf von Aden und den Indischen Ozean im Süden und die Sandwüste des »leeren Viertels« (Rub` alKhali) im Nordosten. Zahlreiche archäologische Stätten und Inschriften zeugen von den antiken Reichen, die hier existierten. Obwohl die Region im Nordosten durch die enorme, fast leere Wüste von Mesopotamien und Levante getrennt war, blieb sie mit diesen Regionen durch mehrere Handelswege verbunden, die vor allem durch die bergige Landschaft des Hijaz führten, wo sich die Stadt Mekka befindet. Heute ist der Großteil dieses Gebiets Staatsgebiet der Republik Jemen.

\section{Historischer Überblick}

Die mittelalterliche Geschichte des Jemen kann in vier Phasen unterteilt werden. ${ }^{1} \mathrm{Ab}$ Mitte des 7. bis Mitte des 9. Jahrhunderts gab es mehrere mächtige unabhängige Dynastien, aber auch die Kalifen der Umayyaden und Abbasiden entsandten Statthalter nach Sanaa und in andere regionale Zentren wie al-Janad. Es ist schwer zu festzustellen, wie viel und welche Art von Einfluß und Macht diese Statthalter wirklich hatten. Das Hochland war noch von Familien der alten Elite dominiert, weshalb die Abbasiden ihre Statthalter oft aus deren Reihen rekrutieren mußten. Im Küstengebiet der Tihama formierte sich die Dynastie der Ziyadiden (819-981) aus Statthaltern der Abbasiden, die jedoch bald mit dem Kalifen brachen.
Ende des 9. Jahrhunderts entwickelten sich mehrere schiitisch geprägte Bewegungen im Hochland, darunter die Zaiditen und Ismailiten. Im Jahre 897 ließ sich der erste zaiditische Imam in der nördlichen Stadt Sa'da nieder. Zusammen mit verbündeten Stämmen nahmen zaiditische Imame in mehreren Feldzügen große Teile des Hochlands, einschließlich Sanaa, ein. Über lange Zeit beschränkten die Zaiditen sich jedoch auf wenige Städte im Norden. Das erste ismailitische Untenehmen war der Feldzug von Ali ibn al-Fadl und Mansur al-Yaman gegen Ende des 9. Jahrhunderts, während dem sie mehrere Male gegen die lokalen Dynastien der Yufiriden (847-997) und der Zaiditen um die Gebiete rund um Sanaa kämpften.

Im Laufe des 11. Jahrhunderts entwickelte sich eine stark regionalisierte politische Landschaft in ganz Südarabien. Die Macht der zaiditischen Imame im Hochland nördlich von Sanaa war stark zersplittert. Die neue ismailitische Dynastie der Sulaihiden (1047-1138) übernahm die Vorherrschaft im zentralen und südlichen Hochland zuerst mit Sanaa als Hauptstadt, später bewegten sie sich nach Süden in die Stadt Jibla, wo die berühmte Königin Arwa etwa fünf Jahrzehnte lang regierte. In der Küstenebene des Roten Meers gründeten zwei abessinische Sklaven die Dynastie der Najahiden (10211156) in der Stadt Zabid. Zur gleichen Zeit kamen die Sulaymaniden (1069-1173) aus Mekka in den Norden der Tihama und gründeten ihre Hauptstadt Harad. An der Küste des Indischen Ozeans entstand in der Stadt Aden eine weitere fatimidische Dynastie, die Zurayiden (1080-1174). Ende des Jahrhunderts übernahmen drei aufeinanderfolgende Dynastien der Hamdaniden (1098-1174) die Region Sanaa und gerieten in Konflikt mit den zaiditischen Imamen. Mitte des 12.
Jahrhunderts eroberten die Mahdiden (1136-1174) das südliche Hochland und die Tihama und bedrohten die Najahiden und Sulaymaniden, die Unterstützung von den Ayyubiden in Ägypten anforderten.

Sowohl als Antwort darauf als auch aus Interesse an der Kontrolle über den Handel im Roten Meer überquerten die Ayyubiden das Rote Meer und eroberten im Jahre 1174 rasch den gesamten Süden bis nach Sanaa, wodurch sie eine neue politische Dynamik in ganz Südarabien schufen. Für die nächsten dreieinhalb Jahrhunderte war die Region in zwei politische Sphären geteilt. Die zaiditischen Imame herrschten weiterhin im nördlichen Hochland, während die Ayyubiden (1174-1228), die Rasuliden (1228-1454) und schließlich die Tahiriden (1454-1517) das südliche Hochland und die Küstengebiete kontrollierten. Die Grenze zog sich grob von Sanaa im Norden bis zum Sumara-Gebirgspaß im Süden, obwohl von beiden Seiten auch darüber hinaus Feldzüge unternommen wurden. Nach einer kurzen Invasion der Mamluken im Jahre 1517, die mit den ersten Feuerwaffen Südarabien einnahmen, übernahmen die Osmanen die Tihama im Jahre 1539 und markierten damit das Ende des Mittelalters.

\section{Stammesgemeinschaften}

Zu den bedeutenden Merkmalen der mittelalterlichen Geschichte Südarabiens zählt neben diesen dynastischen Veränderungen der ständige Kampf zwischen den lokalen Stämmen und oft fremdländischen Staaten um die politische Vorherrschaft in verschiedenen Teilen der Region. ${ }^{2}$ Während die verschiedenen Staaten während der gesamten Zeit immer wieder verschwanden und auftauchten, blieben die Stammesgemeinschaften bestehen. Leider sind diese Gruppen aufgrund der schwierigen Quellenlage oft 


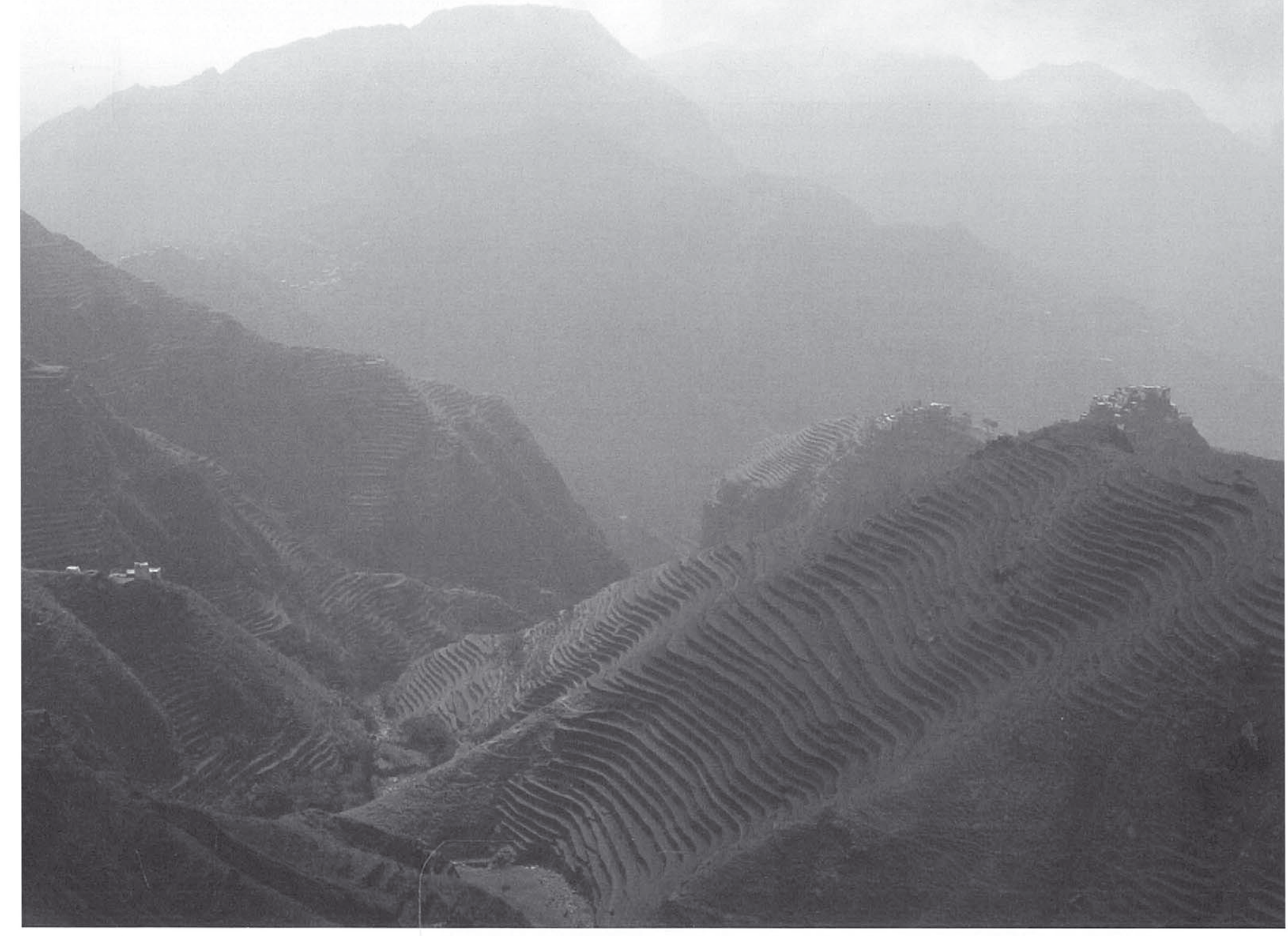

schwer in den historischen Aufzeichnungen erfaßbar, aber einige Aspekte, einschließlich ihrer Genealogie, ihrer geographischen Territorialisierung und oft feindlichen Auseinandersetzungen mit anderen Staaten können untersucht werden.

Mit dem Niedergang des vorislamischen Himyar-Imperiums im 6. Jahrhundert bildete sich eine neue politische Landschaft in Südarabien, in der die zerstreuten Stämme in det gesamten Region wichtige Mitbewerber um die Vorherrschaft wurden. Im Laufe det nächsten Jahrhunderte verließen einige dieser Stämme Südarabien und schlossen sich den islamischen Eroberungen im Irak, Syrien und Ägypten an. Andere blieben und rebellierten gegen die Versuche des neuen Kalifats, die Region zu kontrollieren und Steuern zu erheben. Zur gleichen Zeit in diesem frühen Abschnitt des Mittelalters gewann der Begriff der Verwandtschaft eine größere Bedeutung für die Beschreibung der Beziehungen zwischen den Stämmen. Vorher waren sie vor allem durch ihr Gebiet definiert, es fanden sich nur wenige Quellen zu ihrer Abstammung. Mit diesem neuen Ge- fühl des sozialen Zusammenhalts durch wahrscheinlich fiktive Blutsbande organisierten sie sich als Gruppen mit gleicher Abstammung und gaben sich namengebende Vorfahren. Kahtan (auch: Joktan), ein Nachkomme des Patriarchen Noah, wurde als der Urvater der Südaraber benannt. Sein Pendant in Nordarabien war Adnan, ein Nachkomme des Patriarchen Abraham.

Die beste Darstellung dieses genealogischen Netzwerks im Mittelalter findet sich in dem mehrbändigen Werk Das Diadem aus dem 10. Jahrhundert. ${ }^{3}$ Es wurde von Muhammad al-Hasan al-Hamdani verfaßt, der im nördlichen Hochland lebte und sich mit den neu angekommenen Zaiditen so zerstritt, daß er mehrere Jahre im Gefängnis verbrachte. Seine Unzufriedenheit mit der Art und Weise, wie südarabische Stämme von Genealogen aus dem Norden bis dahin dargestellt wurden, veranlaßte ihn dazu, drei der Bände den Verbindungen zwischen den großen Stammesverbänden der Hamdan, Himyar, Madhhij und Khawlan zu widmen. Durch eine Zusammenstellung von Daten aus mündlichen Quellen und schriftlichen
Shirāqī Hajja im westlichen Bergland. Bild: E. Hovden.

Aufzeichnungen seiner Stammesgenossen und einer genauen Beschreibung der internen Beziehungen der verschiedenen Teile der Stammesgemeinschaft mit einem besonderen Schwerpunkt auf ihrer direkten Verbindung zu den Arabern aus vorislamischer Zeit kommt al-Hasan al-Hamdani in Das Diadem zu dem Schluß, daß durchaus eine Gruppen- oder Stammesidentität besteht. Der Text ist durchsetzt von Gedichten, die von der altertümlichen Kriegsführung erzählen und die Tapferkeit der Helden auf dem Schlachtfeld preisen. Leider sind die meisten dieser Ereignisse nicht datiert, so$\mathrm{da}$ es schwierig ist, sie in eine narrative $\mathrm{Ab}$ folge zu bringen. Dennoch gelingt es diesen genealogischen Texten, eine handfeste ideologische und symbolische Darstellung der Stammesgemeinschaft zu schaffen.

Ein weiterer wichtiger Aspekt der mittelalterlichen Stammesgemeinschaften in Südarabien war ihre geographische Verteilung, 


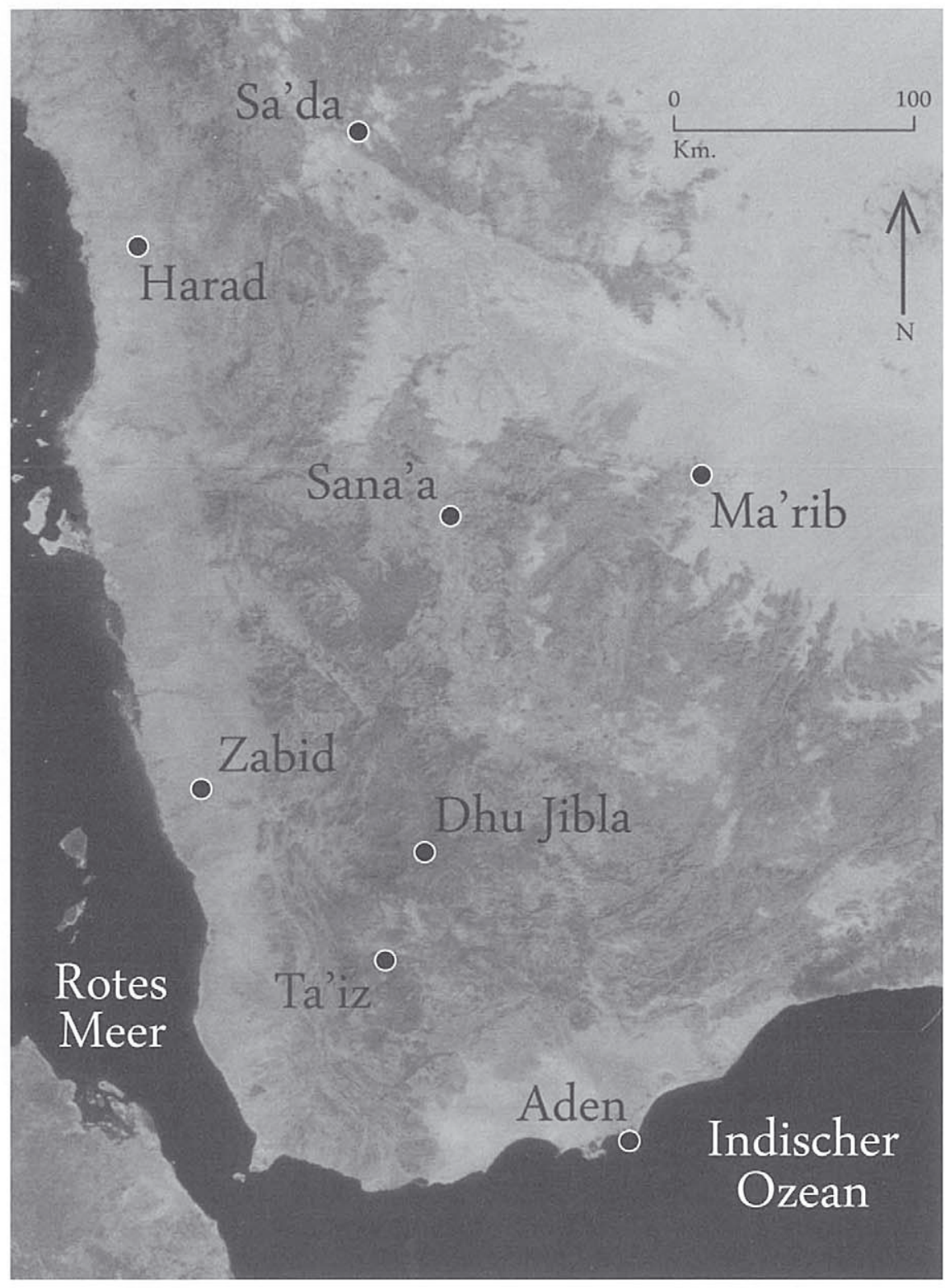

oft verglichen mit einem territorialen Schachbrett. Im Gegensatz zu den Stämmen nomadisch lebender Hirten in anderen Teilen der arabischen Halbinsel bestanden die Stämme in Südarabien aus seßhaften Bauern. Damit wurde das Gebiet, das sie bewohnten und bewirtschafteten, zu einem bevorzugten Mittel, mit dessen Hilfe die Stämme sich selbst wahrnahmen und organisierten. Ein zweiter Text von al-Hamdani, ebenfalls verfaßt im 10. Jahrhundert, ermöglicht die Untersuchung dieses raumbezogenen Merkmals der tribalen Identität. Dieses geographische Werk mit dem Titel Beschreibung der Arabischen Halbinsel ${ }^{4}$ liefert eine umfassende Beschreibung der ganzen Arabischen Halbinsel, stellt jedoch die Region Südarabien deutlich in den Mittelpunkt. Es enthält eine Vielzahl von Informationen über die physiographischen Attribute der
Links: Jemen, westliches Bergland. - Rechts: Wadi in Jabal Rayma im westlichen Bergland. Bild: E. Hovden.

Im Gegensatz zu früheren Autoren, die nicht in Südarabien angesiedelt waren und lediglich eine Liste der verschiedenen mikhlafs zusammenstellen konnten, gab al-Hamdani oft auch die Stämme an, die dort wohnten. Einige mikblafs bestehen nur aus Stämmen, die derselben Konföderation angehörten. Die mikhlaf Dhi Ru'ayn ${ }^{5}$ zum Beispiel, die sich im Kernland des ehemaligen HimyarReichs befindet, enthält erwartungsgemäß nur Himyar-Stämme, während der einzige bekannte Stamm der mikblaf $\mathrm{Ma}^{\prime} \mathrm{rib}^{6}$ in der östlichen Wüste Murad von Kuhlan Madhhij war, der laut Geschichtsforschung seit Jahrhunderten in und nördlich jener Region angesiedelt war. Andere mikeblafs enthalten jedoch Stämme von mehr als einer Konföderation, was auf eine komplexe Interaktion zwischen den Stämmen schließen läßt. Im mikblaf Aqyan $^{7}$, nördlich von Sanaa, gibt es einen Stamm mit Zugehörigkeit zum HimyarStaat und mehreren Stämmen aus der Hamdan-Konföderation. Folglich erscheint alHamdanis Beschreibung dieses mikhlafs als eine Momentaufnahme, die eine Übergangsperiode betrachtet, in der die Macht der Himyariten abnahm, während andere Stämme in ihren früheren Regionen immer mächtiger wurden. So lassen sich anhand der Analyse der Stammesgeographie bedeutende historische Entwicklungen nachvollziehen.

Der in den historischen Quellen meistgenannte Aspekt der Stammesgemeinschaften sind deren angespannte, bisweilen feindliche Beziehungen mit Staaten, die versuchten, sie zu kontrollieren oder auszubeuten. Diese Berichte finden sich in den zahlreichen Chroniken und Biographien, die von Historikern im Dienste des Staates geschrieben wurden. Aus diesem Grund erscheinen sie oft einseitig, aus der Perspektive der Herrschenden, mit der ideologisch gefärbten Botschaft der fortdauernden und gottgewollten Dominanz über die rebellischen Stämme. Die Verwundbarkeit der Staaten wird allerdings gelegentlich auch dargestellt, wenn Niederlagen gegen die Stämme beschrieben werden. Es wird gezeigt, daß diese Aufeinandersetzungen oft sehr vielschichtig waren und $\mathrm{da}$ keine der Seiten die andere vollständig unterwerfen konnte.

Ein gutes Beispiel für diese Dynamik ist einer Chronik zu entnehmen, die sich in an- 


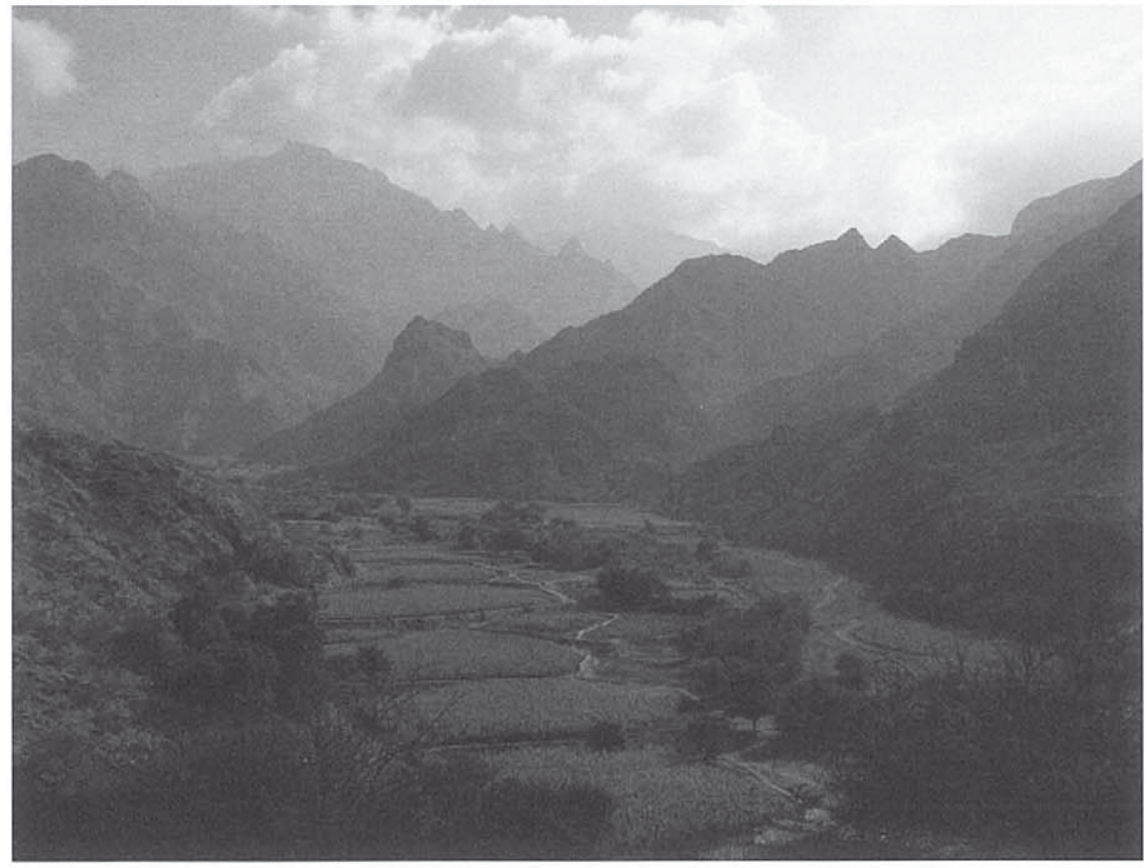

nalistischer Anordnung mit den Ereignissen der ersten sieben Herrscher der RasulidenDynastie bis zum Jahr 1400 befaßt. Sie trägt den Titel Die Perlenketten der Geschichte der Rasulidendynastie ${ }^{8}$ und enthält bedeutende militärische, politische und gesellschaftliche Ereignisse sowie am Ende eines jeden Jahres Todesanzeigen wichtiger Persönlichkeiten. Der Autor Ali ibn al-Hasan al-Khazraji lebte in der Stadt Zabid, einer der Hauptstädte des Rasuliden-Staates, und starb im Jahre 1409. In seinem historiographischen Werk bietet er einen Einblick in die heftigen Auseinandersetzungen zum Beispiel zwischen der Regierung der Rasuliden und den Madhhij-Stämmen im zentralen Hochland. Meist wurden diese Zusammenstöße dadurch ausgelöst, daß ein Rasuliden-Sultan oder ein Prinz eine befestigte Stellung, die von einem örtlichen Stamm besetzt war, einnehmen wollte. Im Jahr 1296 zum Beispiel marschierte Sultan al-Ashraf in die Festung von Mathwa ein, um sie zu sichern, da zuvor siebzig Soldaten und ihre Kommandanten von Stammesangehörigen der Madhhij massakriert worden waren, die das Fort schon für einige Zeit beobachtet hatten. ${ }^{9}$ Im nächsten Jahr belagerte Prinz Saif ad-Din die Festung von Shakab und entriß sie den Händen einer Gruppe Scheichs der Madhhij. In beiden Fällen zeigt die einfache Darstellung des komplexen Wechselspiels, in dem die einzelnen über die Ebene verteilten Festungen besetzt wurden, den anhaltenden Kampf jedes Akteurs, die Macht gegenüber dem anderen in der Region zu sichern. Dieses Muster zeigt sich am deutlichsten in der ausführlichen Beschreibung einer Stammesgruppe aus der Region Jahran, die im Jahre 1272 in der Stadt Dhamar nahezu die gesamte Rasuliden-Garnison auslöschte $^{10}$, und zeigte, daß nicht einmal dieser zentrale Ort für den Staat sicher war. Dies wird noch unterstrichen, als der Sultan al-Muzaffar als Folge davon bei seiner Ankunft in der Stadt den Bau einer Mauer anordnete, um den Besitz der Stadt zu sichern. Auch das bot jedoch keinen ausreichenden Schutz, die Rasuliden zogen sich 1339, weniger als siebzig Jahre später, aus der Region zurück.

\section{Religiöse Gemeinschaften}

In der vorislamischen Vergangenheit waren sowohl Polytheismus als auch Monotheismus, vertreten im Christentum und vor allem im Judentum, bekannt und weit verbreitet. Die wichtigste religiöse Veränderung, die den Beginn des Mittelalters markiert, ist die Einführung des Islam im Jemen im 7. Jahrhundert. Es ist nicht bekannt, wie schnell der Islam in die Kultur der lokalen Bevölkerung integriert wurde, aber er verbreitete sich wahrscheinlich zunächst durch die Eliten und intellektuellen Netzwerke in den Städten.

Überliefert ist jedenfalls, daß der Islam im Jemen sofort angenommen wurde, aber dieser Konvertierungsprozeß muß kritisch gesehen werden, denn man kann nicht davon ausgehen, daß die Gesamtbevölkerung auf Anhieb über das nötige religiöse Wissen verfügte und alle Regeln des Islam befolgte. Es ist nur wenig darüber bekannt, welchen Einfluß der Islam auf die Bevölkerung hatte und inwieweit ältere Formen des Monotheismus oder lokale religiöse Varianten weiterhin praktiziert wurden. Die »Islamisierung« des gesamten Jemen stand sicherlich vor einigen Hürden, sowohl in bezug auf die Einführung der neuen Religion als auch in bezug auf die Durchsetzung eines politischen Systems als Erweiterung des zentralen Kalifats.

Ab etwa der Mitte des 9. Jahrhunderts gibt es Quellen, die von religiösen Führem und ihren Sekretären verfaßt wurden. Ein bekanntes Beispiel ist die Biographie des ersten zaiditischen Imams im Jemen, al-Hadi ila al-Haqq al-Mubin Yahya ibn al-Husayn, die von seinem Mitstreiter Ali ibn $\mathrm{Mu}-$ hammad ibn Ubayd Allah al-Abbasi al-Alawi geschrieben wurde. ${ }^{11}$ Sie beschreibt, wie unwillig einige der Stämme den Islam akzeptierten und wie sie weiterhin ein von Alkohol und sexueller Gastfreundschaft beflecktes, sündhaftes Leben führten. Allerdings ist hier Vorsicht geboten, da diese Quellen zum Großteil geschrieben wurden, um die betreffenden religiösen Führer zu legitimieren und ihre Feinde als zu islamisierende Subjekte darzustellen.

$\mathrm{Zu}$ dieser Zeit gab es auch bedeutende Entwicklungen in den Bereichen Theologie und Recht. Die gemeinsame islamische Kultur vereinte die intellektuellen Eliten mit anderen Regionen der islamischen Welt und vor allem mit religiösen Zentren wie Bagdad, Kairo und Mekka. Die reiche geistige Kultur wurde zu dieser Zeit durch reisende Schüler und Lehrer überliefert, unterstützt durch relativ erschwingliches Papier und Tinte und verbreitet durch Netzwerke religiöser Sekten und Orden. Während der Islam stärker in die Gesellschaft integriert und zu einem wesentlichen Bestandteil des kulturellen und intellektuellen Lebens wurde, begannen auch die Jemeniten, die Religion verstärkt als politisches Instrument einzusetzen. So blieben beispielsweise die Yufiriden im Hochland, die zuvor die Statthalter der Abbasiden abgelehnt und den Großteil des Hochlandes selbst regiert hatten, dem Kalifat im religiösen Sinne treu. Sie hielten das Freitagsgebet im Namen der abbasidischen Kalifen und ließen Münzen prägen, die ihre Namen trugen. $^{12}$

Ende des 9. Jahrhunderts entstanden vor allem im politisch heterogenen Hochland einige schiitisch orientierte Sekten und Netzwerke im Jemen. So bildete sich eine neue Dynamik heraus, in der verschiedene religiöse Sekten gegeneinander antraten und sich in Kriegszeiten sogar gegenseitig als Ungläubige betitelten. Eine dieser Sekten waren die Zaiditen, die zuerst als Gegenbewegung zu den Umayyaden und Abbasiden- 


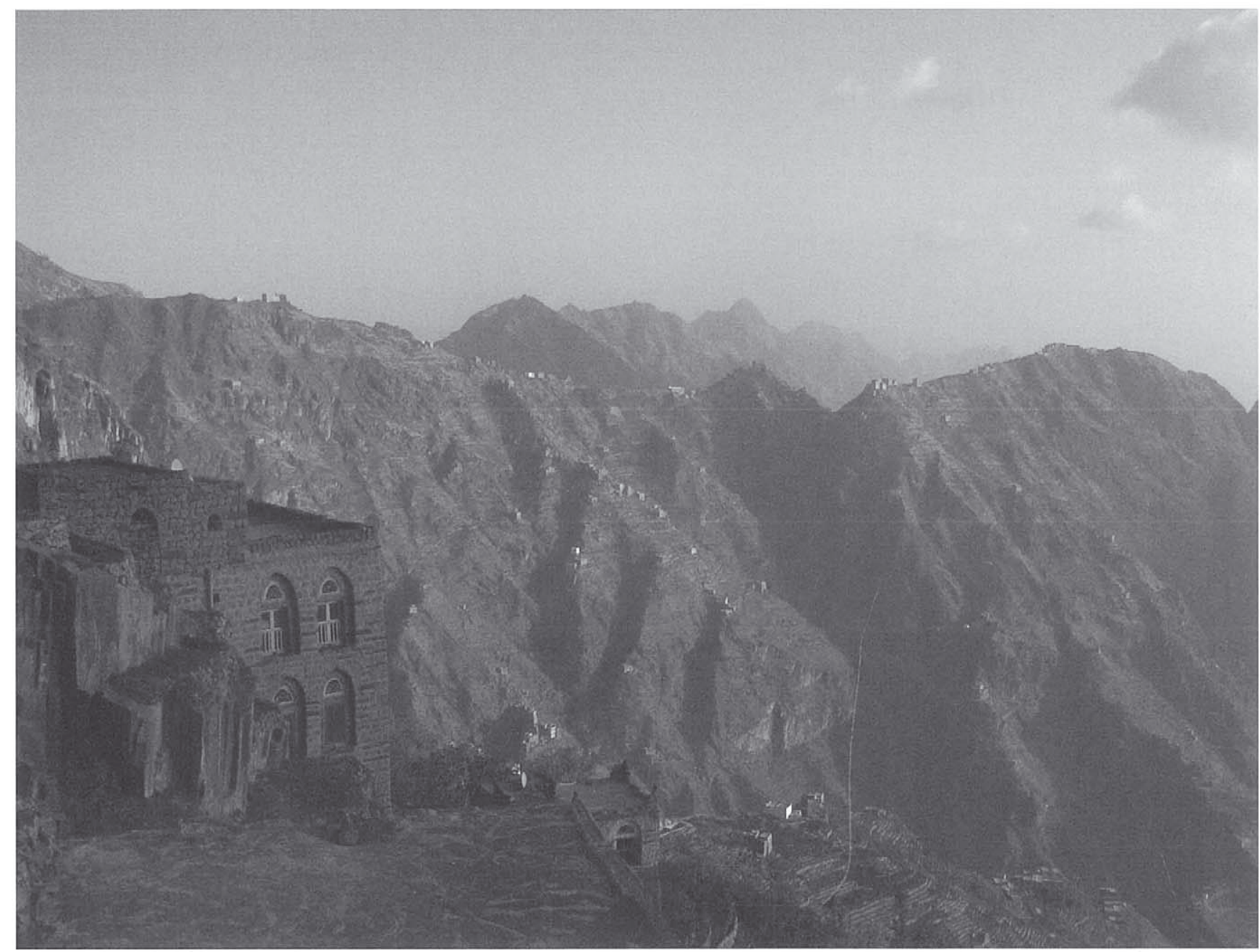

Kalifen entstanden war. Diese Sekte hatte proto-schiitische Merkmale: Den Nachkommen des Propheten wurde eine Sonderstellung in bezug auf religiöse Autorität eingeräumt. Sie wurden »Familie des Hauses [des Propheten]« (Ahl al-bayt) oder Ashraf (Singular Sharif, wobei sharaf Ehre bedeutet) genannt. Während einige der Ashraf politisch in der Gegend um Mekka aktiv waren, versuchten andere im späten 9. Jahrhundert und danach, sich im Stammeshochland nördlich von Sanaa zu etablieren. Thre besondere Form der islamischen Lehre besagte, daß die Führung (Imamat), anders als bei den etablierten Schiiten, nicht erblich war, sondern vielmehr, daß der Anführer (Imam) von den Eliten aus der Gruppe der Ashraf gewählt werden sollte. Wenn ein würdiger Anwärter von den Eliten durch Eid (bay a) gewählt worden war, mußte ihm gehorcht werden, solange er lebte. In der Praxis hatten die meisten Imam-Anwärter keine große politische Macht, aber einige waren dennoch sehr einflußreich, und so nahmen die Zaiditen während des Mittelalters immer größere Teile des nördlichen Hochlands ein, vor allem nach 1323, als die Stadt Sanaa in ihrer Macht war. Die Ashraf als eine religiöse und soziale Gruppe entwickelten eine Art Symbiose mit der Stammesgemeinschaft und den Stämmen. Stammesführer und Zaiditen-Imame waren während des gesamten Mittelalters und auch in späteren Perioden wichtige Akteure der politischen Geschichte des Hochlandes. Die Ashraf verheirateten ihre Töchter jedoch üblicherweise nicht mit der lokalen Stammesbevölkerung und blieben damit eine abgegrenzte Gruppe in der Gemeinschaft der Stämme

Eine weitere schiitische Sekte, die im Hochmittelalter an Bedeutung gewonnen hatte, waren die Ismailiten. Die ismailitischen Fatimiden in Ägypten unterstützten sowohl auf politischer als auch auf religiöser Ebene lokale Vertreter im Jemen, die in kurzer Zeit viele der Festungen in den westlichen Bergen eingenommen hatten. So herrschten die Sulaihiden über große Teile des Hochlandes. Andere ismailitische Dynastien waren Lokaldynastien kleinerer Herrscher in Städten wie Schibam oder Sanaa, wie zum Beispiel die Hatimiden, die die ismailitischen Lehren vertraten, aber den Menschen erlaubten, andere Formen des Islam zu praktizieren, solange sie sich nicht in das politische Geschehen einmischten.

Nach der Invasion der sunnitischen Ayyubiden im Jahre 1174 verschwanden die Ismailiten fast vollständig. Die Zaiditen waren ebenfalls schwach und zerrüttet, aber nach 1323 eroberten sie Sanaa zurück und festigten ihre Macht im südlichen Hochland und im Westen der Stadt. Damals waren die sunnitischen Rasuliden bereits Herrscher im südlichen Hochland und in der Küstenebene rund um die Stadt Zabid. Sie förderten religiöses und intellektuelles Leben durch die Errichtung einer großen Zahl von Religionsschulen (Medresen). Diese waren im wirtschaftlichen Sinne Stiftungen und somit unabhängig. Angehörige der Elite erbauten diese architektonisch vollkommenen Gebäudekomplexe und sorgten dafür, daß die Schulen genügend Landbesitz hatten, um autark sein zu können. Oft gab es Zimmer für Studenten, eigene Wasserversorgung, eine angeschlossene Moschee und feste Gehälter für mehrere Hausmeister und Lehrer. ${ }^{13} \mathrm{Da}$ je- 
Links: al-Ja'fariyya, Rayma, westliches Bergland. - Rechts: Dhu Jibla, nahe Ibb. Bilder: E Hovden.

doch viele dieser Medresen in Stadtzentren lagen, muß davon ausgegangen werden, daß es doch eine enge Verbindung zwischen Lehre und politischer Macht gab. Zu dieser Zeit wurde Zabid nicht nur im Jemen, sondern auch in der restlichen islamischen Welt zu einem angesehenen Zentrum islamischer Lehre.

Im Frühmittelalter waren Christen vor allem in der Stadt Najran angesiedelt, und das gesamte Mittelalter hindurch gab es jüdische Bevölkerung in mehreren Städten im Jemen. Während des gesamten Mittelalters wurde der Handel in nach Ethnien unterteilten Netzwerken getätigt, Beispiele dafür sind die jüdischen Händler im Roten Meer und die indischen Händler, als Banyan bekannt, zwischen Indien und dem Golf von Aden.

Der Zusammenhang zwischen Visionen von Gemeinschaft und Religion ist komplex. Oft wurden politische Ideologie und Loyalitäten mit religiösen Überzeugungen und Lehren vermischt. Ein Zaidi-Muslim zu sein, konnte also bedeuten, sich einfach an die grundsätzlichen Gebetsrituale des islamisch-zaiditischen Rechts zu halten. Es konnte aber auch beinhalten, daß man der zaiditischen politischen Elite loyal gegenübersteht, Steuern zahlt und für sie in den Krieg zieht, auch gegen andere Muslime. Man gehörte also einer rechtlichen und politischen Gemeinschaft an, in der islamischzaiditische Theorien als konzeptionelle, politische und rechtliche Sprache verwendet wurden. Die meisten der größeren regionalen Religionsgemeinschaften im mittelalterlichen Jemen wiesen Elemente dieser beiden Ebenen auf. Die Ebene der Religiosität bezog sich auf die tägliche Ausübung des Glaubens, und die andere Ebene hatte eine eher politische Dimension. Das Mittelalter war für den Jemen eine Zeit historischer Prozesse, in der Religion ein wichtiger Teil, wenn auch nicht die gesamte Antriebskraft war. Gerade in der politischen Zersplitterung der Jahre zwischen 850 und 1300 wechselten die lokalen Eliten und damit oft ganze Stammesgruppen häufig die Seiten, und die geographischen Grenzen zwischen verschiedenen Zweigen des Islam veränderten sich noch schnell. Allerdings bedeutet dies nicht,

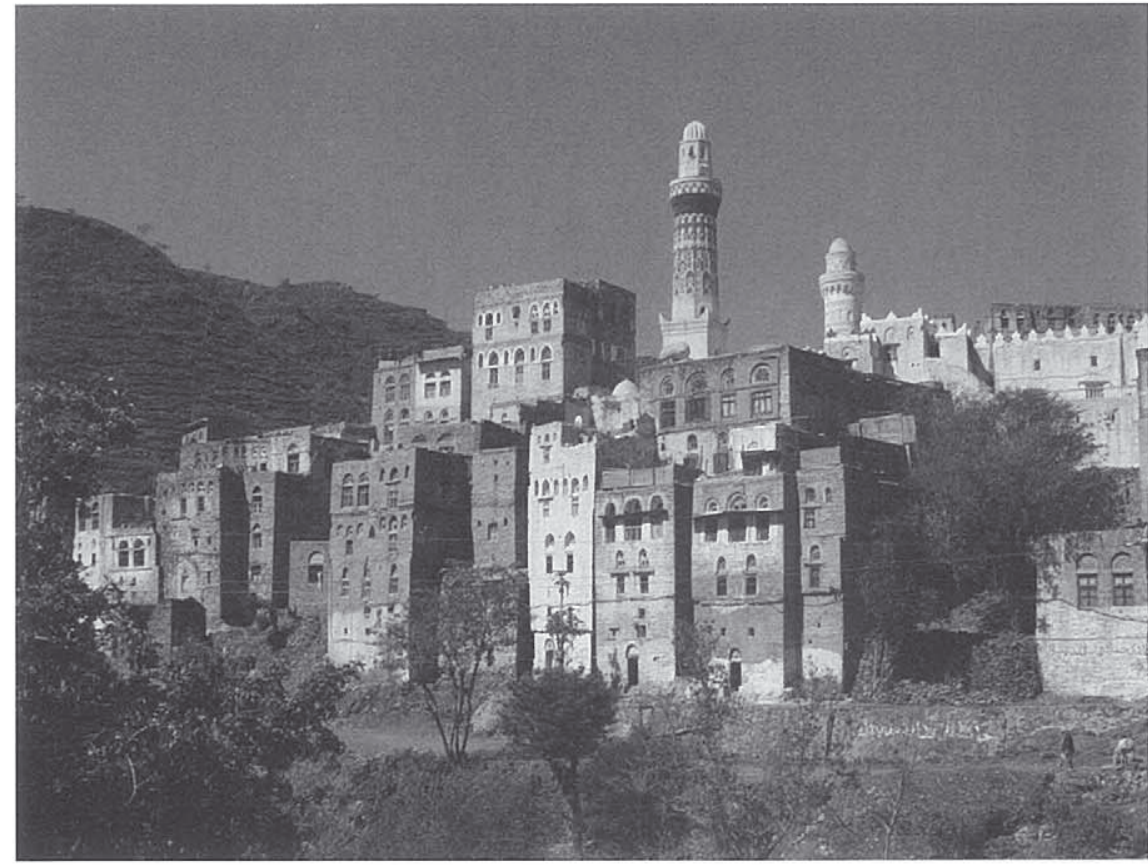

daß sich die Überzeugungen der Bevölkerung im gleichen Tempo veränderten.

Auch für Historiker ist es eine Herausforderung, religiös orientierte Sekten und Bewegungen von denjenigen Herrschern und Dynastien zu trennen, die Führer von Staaten, Stammesallianzen und militärischen Kräften waren und lediglich ein islamisches Vokabular benutzten. Die religiösen Phänomene reichen von Wanderpredigern, die von gelegentlichen Almosen lebten, über millenaristische Bewegungen um charismatische Führer, Netzwerke von gottesfürchtigen und intellektuellen Personen zu länger vorherrschenden religiös-theokratischen Strukturen wie die der Zaiditen in den Hochebenen. Bei der Untersuchung des Mittelalters ist der Blick auf die Religion unvermeidbar - wobei diese sich in der Realität immer anders zeigt als in ihrem Ideal.

\section{Anmerkungen}

1. Weitere Informationen: G. Rex Smith, Politische Geschichte des islamischen Jemen bis zur ersten türkischen Invasion (1-945 Hidschra $=$ 622-1538 n. Chr.), in: Werner Daum (Hg.), Jemen. 3000 Jabre Kunst und Kultur des gliicklichen Arabien, Frankfurt a. M. 1987.

2. Der Begriff Stamm wird hier als Übersetzung für den arabischen Begriff qabäil (s. qabīla) verwendet, der soziale Gruppen bezeichnet. Obwohl der Begriff häufig in der Forschung zur Geschichte des Jemen auftaucht, muß angemerkt werden, daß der Gebrauch des Begriffs Stamm kritisiert wurde, da er einerseits zu stark mit der Zeit des europäischen Kolonialismus verbunden ist und andererseits zu zweideutig ist, um von analytischem Nutzen zu sein. Für eine weiterführende Untersuchung der Stämme im Jemen siehe auch Johann Heiss. Stämme in: Länderkunde Jemen, Horst Kopp (Hg.), Wiesbaden 2005, 51-73. Weiters muß beachtet werden, daß es in einigen Fällen keinen klaren Unterschied zwischen Stamm und Staat gibt. Einige Staaten wurden von Stämmen regiert oder hatten lokale Repräsentanten, die Stammesführer waren. Einige Stämme wendeten staatliche Praktiken wie die Steuererhebung an.

3. al-Hamdānī, al-Ḥasan b. Aḥmad b. Ya quāb. alIkFlil.Muhammad b. 'Alī al-Akwa', (Hg.), Sanaa, 2004 [1979].

4. al-Hamdānī, al-Ḥasan b. Ahmadb. Yá qūb. bifat Jazìrat al'Arab. Muhammad b. 'Alī al-Akwa' (Hg.), Bagdad, 1989.

5. al-Hamdānī, bifat, 200-201.î̀

6. al-Hamdānī, bifat, 203-204.

7. al-Hamdānī, bifat, 211-213.

8. al-Khazrajī, 'Alī ibn al-Hasan. Al-Uqud al Lu'lu' ìyya fí Tāñ̄eh al-Dāwla al-Rasülizya. A. alHibshi, (Hg.), 2 Bände, Sanaa, 2009.

9. al-Khazrajī, al-Uqud, 1:343.

10. al-Khazrajī, al'-Uqud, 1:229.

11. 'Alī b. Muhammad b. 'Ubayd Allāh al-'Abbāsi al-Alawī, Sìrat al-Hādì itā al-Haqq Yabyā b. alHutusayn, Suhayl Zakkār, (Hg.), 2 Ausgabe, Beirut, 1981/1401.

12. Charles L. Geddes. The Yu'firid Dynasty of ban' $a$ ', Unveröffentlichte Doktorarbeit, University of London, 1959, 59.

13. Ismā'īlb. 'Alī al-Akwa, al-Madāris al-istāmiyya fitl-Yaman, Sanaa, Beirut, 1986; 'Abd al-Raḥmān 'Abd 'Allāh al-Haramī, Zabìd: Masäjiduhā waMadärisubā al-Ilmiyya fí al-tārükh, Sanaa, Damaskus, 2000

Eirik Hovden, PhD, eirik.hovden@oeaw.ac.at Daniel Mahoney, PhD, daniel.mahoney@oeaw.ac.at Institut für Sozialanthropologie, Österreichische Akademie der Wissenschaften, Apostelgasse 23, 1030 Wien 


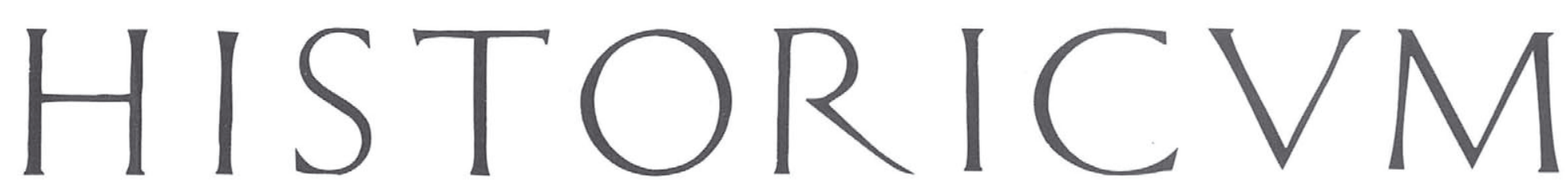

ZEITSCHRIF T UUR GESCHICHTE

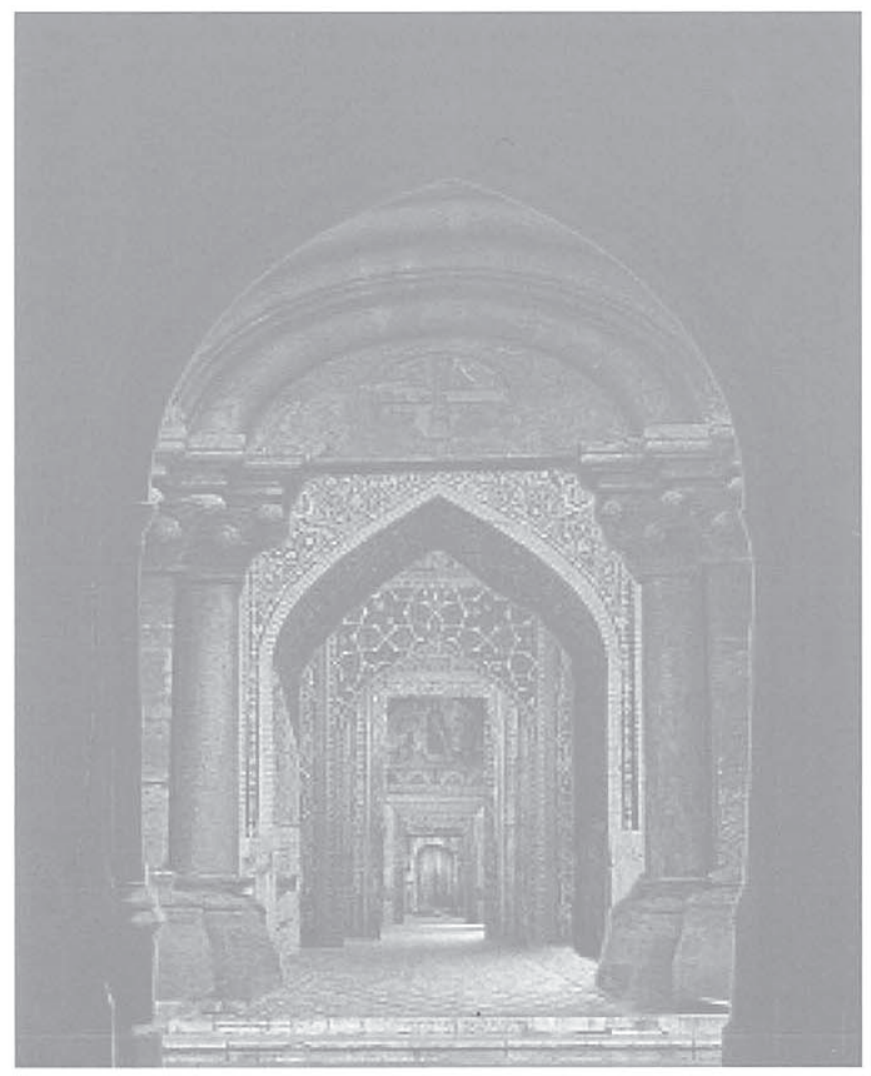

SOMMER - HERBST 20012

VISIONS OF COMMUNITY

$H A B$ I LI T A T I O N

D I R K K R U P P N O W 


\section{Editorial}

Das vorliegende Heft präsentiert Beiträge aus einem interdisziplinären Sonderforschungsbereich des Fonds zur Förderung der wissenschaftlichen Forschung: Mittelalterliche Gemeinschaftsvorstellungen in christlichen, islamischen und buddhistischen Gesellschaften werden dabei vergleichend untersucht. Angesiedelt ist das Projekt an der Österreichischen Akademie der Wissenschaften und an der Universität Wien.

Die Beiträge des Heftes stammen aus einer Ringvorlesung an der Universität Wien, die Gemeinschaftsvorstellungen jeweils mit exklusivem Blick auf eine Gemeinschaft behandelt hat. Der Umfang dieser Gemeinschaften variiert freilich stark und reicht vom christlichen Europa insgesamt bis zu einer einzelnen dalmatinischen Insel. Inkludiert sind Südarabien und Tibet ebenso wie Österreich und das Karolingerreich.

Die Autoren arbeiten überwiegend an

Titelbild: Tore. Corporate Image des Sonderforschungsbereichs "Visions of Community". verschiedenen Instituten der Akademie der Wissenschaften und der Universität Wien: An der Akademie forschen Gerda Heydemann, Veronika Wieser und Rutger Kramer sind (Institut für Mittelalterforschung), Eirik
Hoven und Daniel Mahoney (Institut für Sozialanthropologie) sowie Mathias Fermer (Institut für Kultur- und Geistesgeschichte Asiens, Bereich Tibetologie). An der Universität Wien arbeiten Christian Opitz (Institut für Kunstgeschichte), Elisabeth Gruber (Institut für Geschichte) sowie Fabian Kümmeler und Sascha Attia (Institut für Osteuropäische Geschichte). Irene van Renswoude ist am Institut für kulturwissenschaftliche Forschung der Universität Utrecht im Bereich der Mittelalterforschung tätig.

Dic Teilprojekte von Visions of Community werden von Walter Pohl, Andre Gringrich, Helmut Krasser (von den genannten Instituten der ÖAW) sowie Christina Lutter und Oliver Schmitt (von den Instituten der Universität Wien) geleitet. Rutger Kramer, einer der Autoren dieses Heftes, ist auch Koordinator zwischen den Teilprojekten.

Eine Einführung ins Konzept des Sonderforschungsbereiches gibt die Einleitung von Walter Pohl (Gesamtleiter) zu Beginn dieses Schwerpunkts.

Michael Pammer

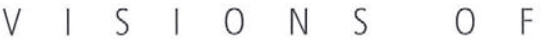 \\ Visions of Community< im interkulturellen Vergleich Von Walter Pohl \\ Gemeinschaftsvorstellungen in Zeiten

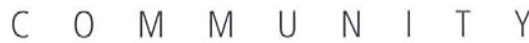 \\ Bilder von Gemeinschaften - Bilder für Gemeinschaften: Zur visuellen Kultur spätmittelalterlicher Dominikanerklöster in Mitteleuropa Von Christian Nikolaus Opitz des Umbruchs: Der Gebrauch der Bibel in Europa zwischen Antike und Frühmittelalter Von Gerda Heydemann und Veronika Wieser

Medieninhaber und Verleger: Aktionsgemeinschaft/ÖSU.Herausgeber:Michael Pammer. Sitz des Verlagesundder Redaktion: Finkengang 27, A4048 Linz-Puchenau. Hersteller: Salzkammergut Media Ges. m. b. H., 4810 Gmunden. Erscheinungsort: Linz. Verlagspostamt: 4040 Linz. Telephon $+43 / 664 / 60246-7000$ Fax $+43 / 732 / 2468-8532$

hup: / |www.wsg-bist.uni-linz.ac.at| Historicum. bom

E-Mail:historicum@jku.at

Konto: PSK (BLZ 60000) 1026.722/HISTORICUM

HISTORICUM dient der Diskussion von Fragen det Geschichtswissenschaft und der Politik. Namentlich gekennzeichnete Beiträge müssen nicht mit der Meinung der Redaktion übereinstimmen.
Dissens, Debatte und Diskurs: Kirche und Imperium in der Karolingerzeit Von Rutger Kramer und Irene van Renswoude

Stammes- und Religionsgemeinschaften im mittelalterlichen Südarabien Von Eirik Hovden und Daniel Mahoney

Tibetische Meister und ihr »befreites Wirken«: Auf Spurensuche monastischer Gemeinschaften in den Lebensgeschichten des mittelalterlichen Tibet Von Mathias Fermer
Städtische Gemeinschaftsbildung im spätmittelalterlichen Herzogtum Österreich

14 Von Elisabeth Gruber

Der Strafe zum Trotz: Gemeinschaft und Konflikt im venezianischen Dalmatien: Ein Blick auf Korčula im 15. Jahrhundert Von Fabian Kümmeler und Sascha Attia

$\begin{array}{llllllllllll}H & A & B & \text { I } & \text { L } & \text { I } & \text { T } & \text { A } & \text { T } & \text { I } & 0 & \mathrm{~N}\end{array}$

28 Dirk Rupnow: 》Judenforschung« im Dritten Reich Von Michael Pammer

Impressum

Ausstellungen 